\title{
Use of Imaging for the Diagnosis of Idiopathic Granulomatous Mastitis: A Clinician's Perspective
}

\author{
Mustafa Hasbahceci1 and Huseyin Kadioglu²
}

\begin{abstract}
Idiopathic granulomatous mastitis (IGM) is an inflammatory breast disease with unknown etiology and nonspecific symptoms. Differentiation of breast cancer from granulomatous disease is a diagnostic and clinical challenge. Imaging features and decision of the surgeon, based on these findings, may be important steps during diagnosis and follow-up period. Articles in association with imaging of IGM were evaluated using a PubMed search in August, 2017. Hypo-echoic or heterogeneous mass/es with or without tubular extensions were the most common ultrasonographic findings. Focal asymmetric density with or without skin thickening and parenchymal distortion were detected in most of the patients during mammography. Magnetic resonance imaging most commonly revealed focal or diffuse asymmetrical signal intensity changes without significant mass effect. Although a specified and standardised criteria list for each type of the imaging modality has been lacking, the most commonly detected imaging findings, especially in cases of multiple lesions in accordance with the decision of the surgeon and/or clinician, can be used for the diagnosis and follow-up of IGM with acceptable safety margins. However, in the presence of any suspicious findings during imaging or clinical examination, histopathological analysis should be performed.
\end{abstract}

Key Words: Granulomatous mastitis, Chronic mastitis, Ultrasonography, Mammography, Magnetic resonance imaging.

\section{INTRODUCTION}

Idiopathic granulomatous mastitis (IGM), as an inflammatory breast disease with unknown etiology, has been defined by Kessler and Wolloch in $1972 .{ }^{1}$ It has been seen that an exact definition of IGM has been lacking, based on the previous publications.2,3 In these studies, IGM has been tried to be defined as a benign, rare and inflammatory lesion with unknown etiology. Therefore, both its definition and diagnostic criteria are still lacking. Due to the presence of nonspecific symptoms including unilateral breast mass, pain, and skin lesions, its diagnosis usually necessitates histopathology to exclude other differential diagnoses. ${ }^{2}$ Development of a skin fistula in a young or premenopausal female patient may not be the characteristic clinical finding for breast cancer, especially in patients from Western countries in which breast cancer develops almost one decade later than the patients living in Asia and Middle East. ${ }^{4}$ Differentiation of IGM from breast cancer or other granulomatous diseases, including tuberculosis, mastitis caused by systemic lupus erythematosus, Wegener's granulomatosis, and sarcoidosis, is a diagnostic and

1 General Surgery Clinic, Medical Park Fatih Hospital, Fatih, Istanbul, Turkey

2 General Surgery Clinic, Istanbul Medicana Hospital, Bahcelievler, Istanbul, Turkey

Correspondence: Dr. Mustafa Hasbahceci, Medical Park Fatih Hospital, Horhor Street No: 4, 34080, Fatih, Istanbul, Turkey E-mail: hasbahceci@yahoo.com

Received: March 02, 2018; Accepted: May 10, 2018 clinical challenge; and should be performed by using imaging and pathological findings.3,5-7 Although this differentiation based on imaging features may be challenging, imaging findings in IGM patients can be well described to help physicians to diagnose the disease. 8 Additionally, the role of the surgeon and/or clinician in decision-making with regard to diagnosis and follow-up of IGM based on the imaging findings, is still to be established. Therefore, the physicians dealing with IGM should be familiar with the imaging findings and the changes in radiological appearances, especially during the follow-up period of IGM. ${ }^{9}$

The purpose of this study was to review imaging findings of IGM based on ultrasonography (US), mammography (MG), and magnetic resonance imaging (MRI).

\section{METHODOLOGY}

For this study, a Pub-Med search was performed in August 2017, using the terms granulamatous, mastitis and imaging, granulamatous, mastitis and ultrasonography, granulamatous, mastitis and mammography, granulamatous, mastitis and magnetic resonance using the filters humans and English. It was seen that the first literature on the subject was in 1994. Therefore, the duration of the literature search was between 1994 and August, 2017. There were 121 publications. After exclusion of the case reports, case series including five patients at the most, and the articles specifically related with elastography and acoustic radiation force impulse imaging, the articles were evaluated with regard to the presence of detailed imaging findings taken by the mentioned modalities. The studies in which imaging 
findings that were reported as only numbers and percentages without detailed imaging features were also excluded. Therefore, the remaining 30 articles with a total of 949 female patients were used for the preparation of this review (Table I).

Ultrasound in IGM: Considering the articles focusing on the imaging findings of IGM via US, it was found that a hypo-echoic or heterogeneous mass/es with/without tubular extensions is the most common finding. $2,5,8,10-20$ Regarding the presence of an inflammatory lesion in the breast tissue, parenchymal heterogeneity and abscess formation in association with the development of a tumoral lesion can be expected to be the cardinal features seen in IGM patients. In Yildiz's study, 83.3\% of the patients were shown to have hypo-echoic masses with tubular connections and increased parenchymal echo pattern. ${ }^{2}$ In Aghajanzadeh's study, which comprised the largest IGM patients in literature, mass formation with several additional features was detected in $78.2 \%$ of the patients by US. ${ }^{21}$ Although the incidence of each finding has varied from one study to another; generally, it has been reported that formation of a mass in association with one of these findings was shown to be detected in almost $60 \%$ to $70 \%$ of the patients. ${ }^{11}$
However, there was a great variability with regard to these rates. In Kiyak' study, abscess formation and parenchymal heterogeneity in association with a mass was found to be present only in one out of 24 patients $(4.2 \%) .^{22}$ In another study, it was shown that there is only parenchymal edema and generalised soft tissue thickening without any discrete lesion beneath the clinically palpable breast tissue in $40 \%$ of the patients. ${ }^{9}$ Some authors also reported different rates for combination of several findings, i.e. parenchymal heterogeneity and indistinct border or focal breast edema and skin thickening. ${ }^{11}$

Due to the heterogeneity and non-standardised parameters, i.e. increased parenchymal heterogeneity or focally decreased echogeneity with acoustic shadowing or parenchymal distortion with acoustic shadowing during reporting ultrasonographic imaging findings, an accurate evaluation based on these results cannot be performed.2,12,21,23,24 Therefore, it has been thought that these features favour an inflammatory process though histopathology is still essential to establish the diagnosis. 10

Although irregularity has been thought to be a marker for malignancy, there was no clear association between

Table I: Studies with their reference number including demographic data and diagnostic techniques.

\begin{tabular}{|c|c|c|c|c|c|c|}
\hline $\begin{array}{l}\text { Reference } \\
\text { number }\end{array}$ & $\begin{array}{l}\text { Number of } \\
\text { patients }(n)\end{array}$ & $\begin{array}{c}\text { Age (year) } \\
\text { (mean } \pm \mathrm{SD} / \text { median) }\end{array}$ & Range (year) & $\begin{array}{l}\text { US } \\
\mathrm{n}(\%)\end{array}$ & $\begin{array}{c}\text { MG } \\
\mathrm{n}(\%)\end{array}$ & $\begin{array}{c}\mathrm{MRI} \\
\mathrm{n}(\%)\end{array}$ \\
\hline 2 & 30 & 33 & $21-50$ & $30(100)$ & $11(36.7)$ & $5(16.7)$ \\
\hline 5 & 11 & 38.7 & $29-61$ & $11(100)$ & $11(100)$ & $9(81.8)$ \\
\hline 6 & 41 & $34 \beta$ & -- & $29(70.7)$ & 29 (70.7) & -- \\
\hline 7 & 8 & 37 & $27-78$ & $8(100)$ & $6(75)$ & $4(50)$ \\
\hline 8 & 27 & 38 & $21-73$ & $26(96.3)$ & 19 (70.4) & -- \\
\hline 9 & 10 & 35.2 & $24-48$ & $10(100)$ & $4(40)$ & $4(40)$ \\
\hline 11 & 48 & -- & -- & $48(100)$ & -- & -- \\
\hline 12 & 54 & 33.1 & $22-44$ & $54(100)$ & $45(83.3)$ & -- \\
\hline 13 & 36 & 37 & $21-51$ & $36(100)$ & $18(50)$ & $36(100)$ \\
\hline 14 & 29 & $35.14 \pm 9.9$ & $20-69$ & $29(100)$ & $14(48.3)$ & $29(100)$ \\
\hline 15 & 20 & 38 & $25-58$ & -- & -- & $20(100)$ \\
\hline 16 & 11 & 38 & $27-53$ & $11(100)$ & $9(81.8)$ & $7(63.6)$ \\
\hline 17 & 43 & 33.5 & $24-49$ & $39(90.7)$ & -- & -- \\
\hline 18 & 43 & 34 & $22-47$ & $22(51.2)$ & $6(14)$ & -- \\
\hline 19 & 15 & 36 & $24-59$ & $15(100)$ & $15(100)$ & -- \\
\hline 20 & 14 & $46 \pm 12$ & $27-78$ & $25(67.6)$ & -- & $1(3)$ \\
\hline 21 & 206 & 32 & $22-40$ & $206(100)$ & $186(60.3)$ & -- \\
\hline 22 & 24 & 38.4 & $28-60$ & $24(100)$ & $7(29.2)$ & $1(4.2)$ \\
\hline 23 & 21 & $36.3 \pm 11.4$ & $20-67$ & $21(100)$ & $19(90.5)$ & $6(28.6)$ \\
\hline 24 & 9 & 45.2 & $35-57$ & $9(100)$ & $9(100)$ & $9(100)$ \\
\hline 25 & 40 & $39.1 \pm 11.5$ & $21-71$ & $40(100)$ & $20(50)$ & $20(50)$ \\
\hline 26 & 17 & 44 & $25-72$ & 15 (88.2) & $16(94.1)$ & $5(29.4)$ \\
\hline 27 & 11 & 34.8 & $19-42$ & $11(100)$ & 10 (90.9) & $2(18.2)$ \\
\hline 28 & 20 & 38.1 & $19-66$ & $12(60)$ & $20(100)$ & - \\
\hline 29 & 27 & $37.81 \pm 7.1$ & $24-56$ & $27(100)$ & $9(33.3)$ & -- \\
\hline 30 & 16 & $34 \beta$ & $24-51$ & $16(100)$ & $9(56.3)$ & $4(25)$ \\
\hline 33 & 39 & 40.33 & $24-58$ & -- & -- & $39(100)$ \\
\hline 34 & 37 & $36 \pm 8$ & $20-67$ & -- & -- & $37(100)$ \\
\hline 35 & 7 & 35 & $28-41$ & $7(100)$ & $3(42.9)$ & $7(100)$ \\
\hline 36 & 12 & 38 & -- & -- & -- & $12(100)$ \\
\hline
\end{tabular}

קmedian; $S D=$ Standard deviation; $\quad$ US = Ultrasonography; $\quad M G=$ Mammography; $\quad$ MRI = Magnetic resonance imaging. 
irregularity of the lesions detected via US and the absence of IGM. ${ }^{2}$ Several studies have reported that presence of a mass with irregular borders is the most common finding with a rate of $47.5 \%$ in IGM patients. ${ }^{25}$ In Atak's study, presence of a mass with irregular borders necessitated the histopathological analysis. Although there was no malignancy in their series, they thought that histopathological evaluation is still essential for differentiation of IGM from malignant pathologies. 25 Therefore, prospective studies are required to clarify the possible association between irregularity of suspicious masses and IGM.

Unilateral axillary lymph node involvement is another important finding in IGM patients. It has been reported that unilateral axillary involvement with concentric mild cortical thickening and preservation of hilus can be seen
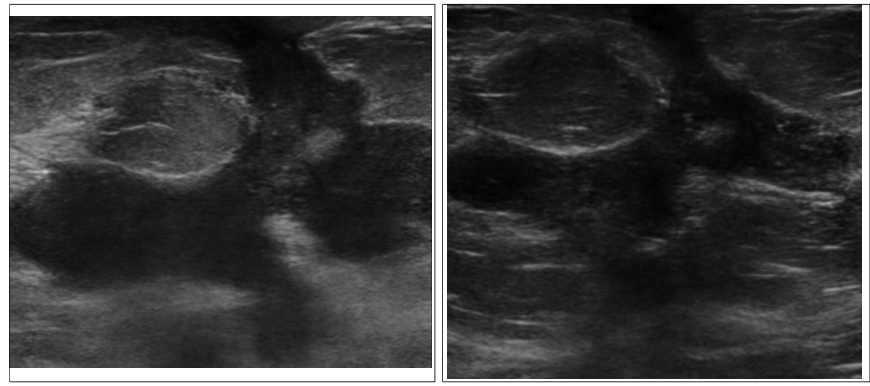

Figure 1 and 2: In the affected breast parenchyma, multiple irregular hypoechoic masses and collections with tubular connections with finger-like aspects, and fistula formation.

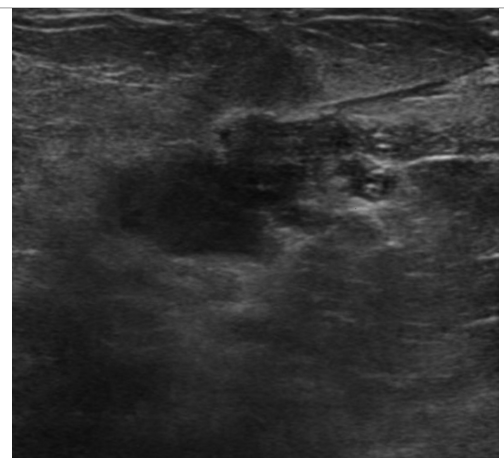

Figure 3: Collection areas with low-level internal echoes consistent with abscess formation. in up to $70 \%$ of the patients. $2,5,11,12,26$ However, other studies have shown that this occurrence is a rare event with a rate of $13.5 \% .21$

Detection of fistula and skin thickening via US is another important imaging finding for IGM. Although these are clinical features which can be detected by physical examination, it is possible to show fistula and skin thickening in almost half or even in $91.2 \%$ of the patients. 2,12,13,27 Contrary to these findings, it has been shown that skin thickening and edema are present in 9 out of 206 patients with a rate of $4.5 \% .{ }^{21}$ Increased vascularity has also been shown to present in up to $75 \%$ of the patients by Doppler US examination. 24,28 Evaluation of elastic features by using sonoelastography has also been studied to reach more accurate diagnosis.11,29 Although no difference between the elastic diameters measured grey-scale and sonoelastagram images was detected, it has also been proposed that a statistically difference has been shown between IGM and malignant breast tissues based on the shear wave velocity marginal and internal values. ${ }^{11,29}$ Therefore, the authors proposed that combination of tissue imaging and tissue quantification techniques via sonoelastography may have a potential to increase the specificity of US to diagnose IGM.11

Based on this data, the characteristic imaging findings of IGM via US has been lacking. Although the physicians may suspect about the diagnosis of IGM using US, there

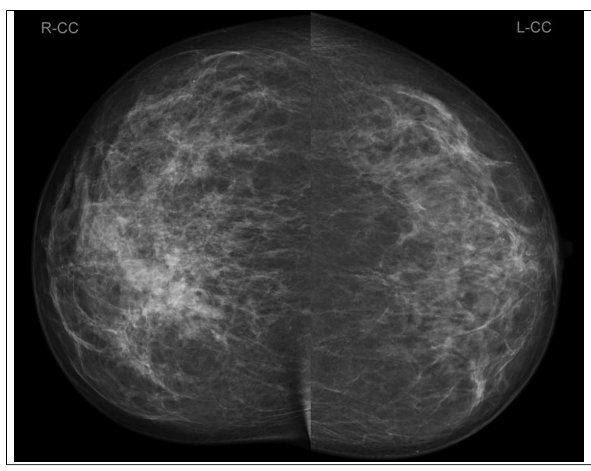

Figure 6: Asymmetric density at right inner quadrant of the right breast.
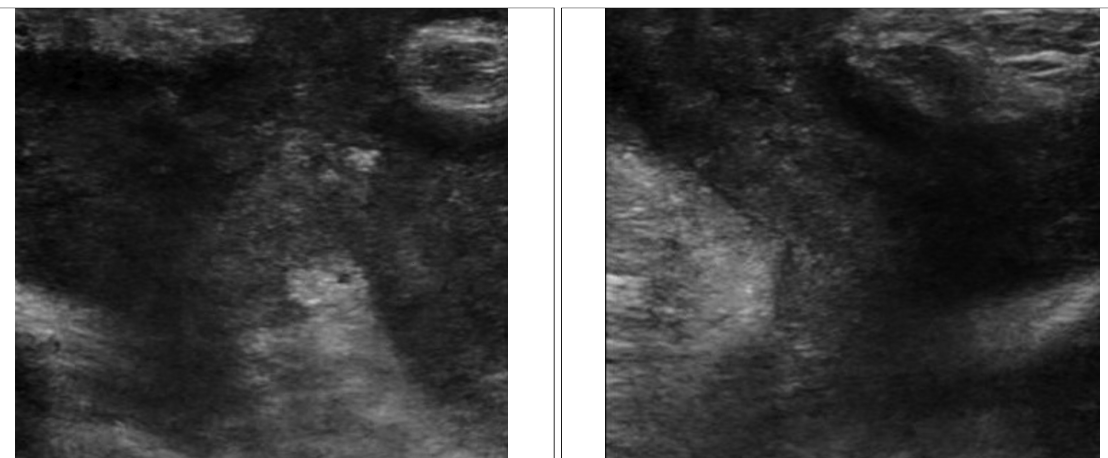

Figure 4 and 5: Increased echo in the affected breast parenchyma with tubular connection with finger-like aspects, and fistula formation.

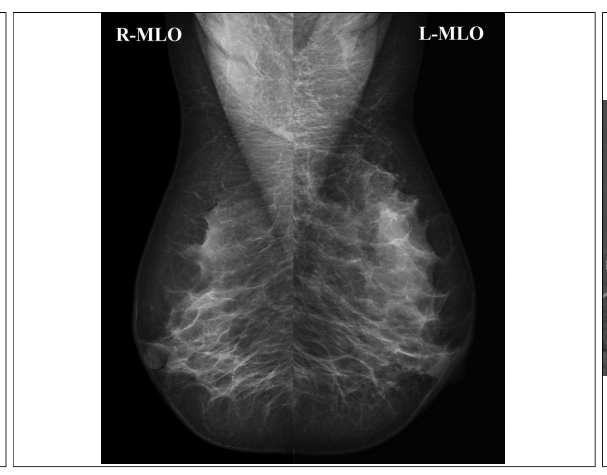

Figure 7: Asymmetric opacity at the upper quadrant of the left breast.

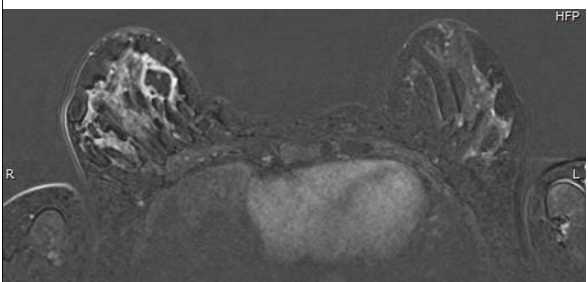

Figure 8: Contrast enhanced T1-w images showing moderate contrast enhancement of the breast parenchyma surrounding hyperintense collections at the right breast. 
should be more specified and standardised criteria to reach more accurate evaluation of these patients.

Mammography in IGM: Because of the more common occurrence of IGM in young female patients less than 35 years of age, radiological evaluation of IGM via MG is less frequently required. In addition, MG usually has less diagnostic power due to overlapping dense breast pattern of young patients obscuring potential findings. ${ }^{23,26}$ Therefore, the data with regard to the use and impact of MG in IGM has been partially restricted in comparison to US.

The most frequent mammographic findings were shown to be focal asymmetric density with/without skin thickening, parenchymal distortion and diffuse asymmetric opacity with trabecular thickening. 2,12,14,22 Aghajanzadeh et al. reported that an irregular focal mass is present in $63.5 \%$ of the patients. Heterogeneous asymmetric density and an irregular or lobulated mass were shown to be detected in $8.5 \%$ and $3.5 \%$ of the cases, respectively. 21 Presence of no abnormality during MG has been a more common event for patients with IGM comparing with US. So, normal mammographic reports have been detected with varying frequencies, up to the half of the patients. ${ }^{2,5,8,13}$ Contrary to this data, dense or extremely dense parenchymal breast pattern was present only in 8 out 186 patients (4.5\%) in Aghajanzadeh's study. ${ }^{21}$ In the light of these controversies, it should be mentioned that there is some conflicting terminology with regard to definition of the focal mass or normal MG results in this study.

It has been reported that suspicious microcalcifications are not usually present in association with IGM.5,13-15,24,27,28 In accordance with ultrasonographic evaluation, axillary lymphadenopathy and skin thickening were also less frequently detected. $8,21,30$

After the comprehensive review of the data taken by MG evaluation of IGM, it is clear that MG cannot be used to diagnose the disease because of the non-specific nature of the mammographic features. Detection of focal or diffuse asymmetric density, parenchymal distortion or an irregular focal mass via MG should necessitate histopathologic evaluation.

Magnetic resonance imaging in IGM: The usefulness of MRI for differentiation of IGM from breast cancer has been questioned previously by several authors. Rieber et al. and deBazelaire et al. showed that MRI cannot differentiate any type of mastitis from inflammatory carcinoma, based on the fact that the nature of both pathologies is inflammatory. 31,32 Detailed analysis of MRI images revealed that focal or diffuse asymmetrical signal intensity changes - hypointense on T1W images and hyperintense on T2W images - without significant mass effect are the most frequent findings. 14,24,33 It has been shown that masses with rim enhancement or clustered-ring non-mass lesions with segmental distribution on MRI are the most commonly detected imaging findings during contrast enhanced MRI. 15,16,24,34 It has been also reported that IGM is seen as non-masslike lesions with restricted diffusion. 33 There was also a wide variation of time intensity curves differing from one patient to another and from lesion to lesion.24 These conflicting results may originate from the fact that there has been limited number of the studies focusing on imaging of IGM via MRI. Additionally, total number of the cases in these studies was very small.7,9,23,26,30,34-36 The authors concluded that there was no characteristic imaging findings due to the wide spectrum of imaging findings of IGM; and non-enhanced or diffusion weighted MRI is not helpful to differentiate IGM from malignant lesions.24,33,34 MRI should not be a cause of delay in histopathological diagnosis of suspected cases. ${ }^{14}$ Therefore, use of MRI for the evaluation of IGM can be recommended in selected case, i.e. the follow-up of biopsy proven IGM, to evaluate the success of conservative treatment or the extent of inflammatory process and to diagnose coexisting inflammatory carcinoma.5,15,31 Additionally, MRI may give some more information in cases with edematous parenchyma preventing detection of lesions by US and MG and dense breast parenchyma in young patients. ${ }^{14}$

Limitations: In the presence of heterogeneity with regard to the parameters used for the diagnosis of IGM, based on the radiological definitions, and small number of the patients in most of the articles were the main limitations. Although there have been many case reports and small case series in the literature, there were limited number of the studies with great number of IGM cases and the results of all diagnostic imaging modalities applied to all cases. Therefore, it could not be possible to perform a systematic review or meta-analysis.

Clinician's perspective in IGM: At that point, imaging modalities with the findings summarised below can be used for the diagnosis of IGM with acceptable safety margins by the clinicians.

\section{A. Ultrasonographic evaluation yielding:}

i. Hypo-echoic or heterogeneous mass/es with/without tubular extensions (Figures 1 and 2).

ii. Hypo-echoic mass with irregular borders.

iii. Parenchymal heterogeneity in association with an abscess (Figure 3).

iv. Fistula tract formation extending to the skin surface (Figures 4 and 5).

v. Reactive appearing enlarged nodes in the ipsilateral axilla in association with hypo-echoic mass/es featured by indistinct, irregular or angular margins, hyper-echoic rim and internal vascularity.

\section{B. Mammographic evaluation yielding:}

i. Focal asymmetric density with/without skin thickening and parenchymal distortion (Figures 6 and 7).

ii. Diffuse asymmetric opacity with trabecular thickening iii. irregular focal mass. 


\section{Magnetic resonance imaging yielding:}

i. Focal or diffuse asymmetrical signal intensity changes without significant mass effect (Figure 8).

ii. Masses with rim enhancement.

iii. Clustered-ring non-mass lesions with segmental distribution.

Although this differentiation, based on imaging features, may be challenging, the physicians dealing with IGM should be familiar with these imaging findings and the changes in radiological appearances, especially during the follow-up period of IGM. The most commonly detected imaging findings, especially in cases of multiple lesions in accordance with the decision of the surgeon and/or clinician can be used for the diagnosis and follow-up of IGM with acceptable safety margins. However, in the presence of any suspicious findings during imaging or clinical examination, histopathological analysis should be performed.

\section{CONCLUSION}

After detailed evaluation of the articles in relation with the imaging features of IGM, it has been understood that there has been great heterogeneity of the articles with regard to the parameters used for this purpose. The radiological definitions of the pathological lesions have varied from one study to another or radiologist to another. In addition, the ranges for the rates used to define the occurrence of any imaging feature have been very wide to reach a consensus. Therefore, there should be a specified and standardised criteria list for each type of the imaging modality for the diagnosis of IGM. As a second step, controlled studies, using these standardised criteria list for the patients with inflammatory breast lesions, should be designed to find sensitivity, specificity, and accuracy of these criteria for IGM. However, in the presence of any suspicious findings during imaging or clinical examination, histopathological analysis should be performed.

\section{REFERENCES}

1. Kessler E, Wolloch Y. Granulomatous mastitis: a lesion clinically simulating carcinoma. Am J Clin Pathol 1972; 58:642-6.

2. Yildiz S, Aralasmak A, Kadioglu H, Toprak H, Yetis H, Gucin Z, et al. Radiologic findings of idiopathic granulomatous mastitis. Med Ultrason 2015; 17:39-44.

3. Azlina AF, Ariza Z, Arni T, Hisham AN. Chronic granulomatous mastitis: diagnostic and therapeutic considerations. World $\mathrm{J}$ Surg 2003; 27:515-8.

4. Song QK, Li J, Huang R, Fan JH, Zheng RS, Zhang BN, et al. Age of diagnosis of breast cancer in China: almost 10 years earlier than in the United States and the European Union. Asian Pac J Cancer Prev 2014; 15:10021-5.

5. Gautier N, Lalonde L, Tran-Thanh D, El Khoury M, David J, Labelle $\mathrm{M}$, et al. Chronic granulomatous mastitis: Imaging, pathology and management. Eur J Radiol 2013; 82:e165-75.

6. Mohammed S, Statz A, Lacross JS, Lassinger BK, Contreras A,
Gutierrez C, et al. Granulomatous mastitis: a 10-year experience from a large inner city county hospital. J Surg Res 2013; 184:299-303.

7. Ozel L, Unal A, Unal E, Kara M, Erdogdu E, Krand O, et al. Granulomatous mastitis: is it an autoimmune disease? Diagnostic and therapeutic dilemmas. Surg Today 2012; 42: 729-33.

8. Handa P, Leibman AJ, Sun D, Abadi M, Goldberg A. Granulomatous mastitis: changing clinical and imaging features with image-guided biopsy correlation. Eur Radiol 2014; 24:2404-11.

9. Bilal A, Badar Albadar F, Bashir Barlas N. Granulomatous Mastitis: Imaging of Temporal Evolution. Scientifica (Cairo) 2016; 2016:3737528.

10. Sripathi S, Ayachit A, Bala A, Kadavigere R, Kumar S. Idiopathic granulomatous mastitis: a diagnostic dilemma for the breast radiologist. Insights Imaging 2016; 7:523-9.

11. Teke M, Teke F, Alan B, Türkoglu A, Hamidi C, Göya C, et al. Differential diagnosis of idiopathic granulomatous mastitis and breast cancer using acoustic radiation force impulse imaging. J Med Ultrason (2001) 2017; 44:109-15.

12. Hovanessian Larsen LJ, Peyvandi B, Klipfel N, Grant E, lyengar $\mathrm{G}$. Granulomatous lobular mastitis: imaging, diagnosis, and treatment. AJR Am J Roentgenol 2009; 193:574-81.

13. Dursun M, Yilmaz S, Yahyayev A, Salmaslioglu A, Yavuz E, Igci $A$, et al. Multimodality imaging features of idiopathic granulomatous mastitis: outcome of 12 years of experience. Radiol Med 2012; 117:529-38.

14. Oztekin PS, Durhan G, Nercis Kosar P, Erel S, Hucumenoglu S. Imaging Findings in Patients with Granulomatous Mastitis. Iran J Radiol 2016; 13:e33900.

15. Poyraz N, Emlik GD, Batur A, Gundes E, Keskin S. Magnetic resonance imaging features of idiopathic granulomatous mastitis: A retrospective analysis. Iran J Radiol 2016; 13: e20873.

16. Al-Khawari HA, Al-Manfouhi HA, Madda JP, Kovacs A, Sheikh M, Roberts O. Radiologic features of granulomatous mastitis. Breast J 2011; 17:645-50.

17. Omranipour R, Mohammadi SF, Samimi P. Idiopathic granulomatous lobular mastitis - report of 43 cases from Iran; introducing a preliminary clinical practice guideline. Breast Care (Basel) 2013; 8:439-43.

18. Kok KY, Telisinghe PU. Granulomatous mastitis: presentation, treatment and outcome in 43 patients. Surgeon 2010; 8:197-201.

19. Memis A, Bilgen I, Ustun EE, Ozdemir N, Erhan Y, Kapkac M. Granulomatous mastitis: imaging findings with histopathologic correlation. Clin Radiol 2002; 57;1001-6.

20. Cheng L, Reddy V, Solmos G, Watkins L, Cimbaluk D, Bitterman $\mathrm{P}$, et al. Mastitis, a radiographic, clinical, and histopathologic review. Breast J 2015; 21;403-9.

21. Aghajanzadeh M, Hassanzadeh R, Alizadeh Sefat S, Alavi A, Hemmati $\mathrm{H}$, Esmaeili Delshad MS, et al. Granulomatous mastitis: Presentations, diagnosis, treatment and outcome in 206 patients from the north of Iran. Breast 2015; 24:456-60.

22. Kiyak G, Dumlu EG, Kilinc I, Tokaç M, Akbaba S, Gurer A, et al. Management of idiopathic granulomatous mastitis: dilemmas in diagnosis and treatment. BMC Surg 2014; 14:66.

23. Akcan A, Akyildiz H, Deneme MA, Akgun H, Aritas $Y$. 
Granulomatous lobular mastitis: a complex diagnostic and therapeutic problem. World J Surg 2006; 30:1403-9.

24. Ozturk M, Mavili E, Kahriman G, Akcan AC, Ozturk F. Granulomatous mastitis: radiological findings. Acta Radiol 2007; 48:150-5.

25. Atak T, Sagiroglu J, Eren T, Ali Özemir I, Alimoglu O. Strategies to treat idiopathic granulomatous mastitis: retrospective analysis of 40 patients. Breast Dis 2015; 35:19-24.

26. Fazzio RT, Shah SS, Sandhu NP, Glazebrook KN. Idiopathic granulomatous mastitis: imaging update and review. Insights Imaging 2016; 7:531-9.

27. Lee JH, Oh KK, Kim EK, Kwack KS, Jung WH, Lee HK. Radiologic and clinical features of idiopathic granulomatous lobular mastitis mimicking advanced breast cancer. Yonsei Med J 2006; 47:78-84.

28. Boufettal H, Essodegui F, Noun M, Hermas S, Samouh N. Idiopathic granulomatous mastitis: a report of twenty cases. Diagn Interv Imaging 2012; 93:586-96.

29. Durur-Karakaya A, Durur-Subasi I, Akcay MN, Sipal S, Guvendi B. Sonoelastography findings for idiopathic granulomatous mastitis. Jpn J Radiol 2015; 33:33-8.

30. Ocal K, Dag A, Turkmenoglu O, Kara T, Seyit H, Konca K.
Granulomatous mastitis: clinical, pathological features, and management. Breast J 2010; 16:176-82.

31. Rieber A, Tomczak RJ, Mergo PJ, Wenzel V, Zeitler $H$, Brambs HJ. MRI of the breast in the differential diagnosis of mastitis versus inflammatory carcinoma and follow-up. J Comput Assist Tomogr 1997; 21:128-32.

32. de Bazelaire C, Groheux D, Chapellier M, Sabatier F, Scémama A, Pluvinage A. Breast inflammation: indications for MRI and PET-CT. Diagn Interv Imaging 2012; 93:104-15.

33. Aslan H, Pourbagher A, Colakoglu T. Idiopathic granulomatous mastitis: magnetic resonance imaging findings with diffusion MRI. Acta Radiol 2016; 57:796-801.

34. Yilmaz R, Demir AA, Kaplan A, Sahin D, Ozkurt E, Dursun M, et al. Magnetic resonance imaging features of idiopathic granulomatous mastitis: is there any contribution of diffusionweighted imaging in the differential diagnosis? Radiol Med 2016; 121:857-66.

35. Kocaoglu M, Somuncu I, Ors F, Bulakbasi N, Tayfun C, Ilkbahar S. Imaging findings in idiopathic granulomatous mastitis. A review with emphasis on magnetic resonance imaging. J Comput Assist Tomogr 2004; 28:635-41.

36. Chu AN, Seiler SJ, Hayes JC, Wooldridge R, Porembka JH. Magnetic resonance imaging characteristics of granulomatous mastitis. Clin Imaging 2017; 43:199-201. 